\title{
Connection between noise and quantum correlations in a double quantum dot
}

\author{
F. Bodoky \\ Kavli Institute of NanoScience, Delft University of Technology, Lorentzweg 1, 2628 CJ Delft, The Netherlands \\ W. Belzig \\ Fachbereich Physik, Universität Konstanz, 78457 Konstanz, Germany \\ C. Bruder \\ Department of Physics, University of Basel, Klingelbergstrasse 82, 4056 Basel, Switzerland
}

(Received 25 September 2007; published 2 January 2008)

\begin{abstract}
We investigate the current and noise characteristics of a double quantum dot system. The strong correlations induced by the Coulomb interaction and the Pauli principle create entangled two-electron states and lead to signatures in the transport properties. We show that the interaction parameter $\phi$, which measures the admixture of the double-occupancy contribution to the singlet state and thus the degree of entanglement, can be directly accessed through the Fano factor of super-Poissonian shot noise.
\end{abstract}

DOI: 10.1103/PhysRevB.77.035302

PACS number(s): 72.70. $+\mathrm{m}, 73.23 .-\mathrm{b}$

\section{INTRODUCTION}

Quantum dots and their current and noise properties ${ }^{1,2}$ are widely investigated topics in today's nanophysics with a number of possible applications, the most revolutionary being a spin-based quantum computer. ${ }^{3}$ Here, the spin of an electron in a quantum dot is used as a qubit, which serves as the basic building block of a quantum computer. A necessary ingredient for quantum processing is the possibility to couple at least two qubits and to understand and manipulate the various correlations. This is one of the motivations to study double quantum dots. ${ }^{4-13}$ In particular, it is required to create entangled electron states by the interaction of an electron inside the double dot with an electron tunneling onto the double dot. Measuring this entanglement is an important experimental task, and theoretical suggestions on how to probe these states are needed.

In this work, we will discuss the relation between zerofrequency current noise, Coulomb correlations, and entanglement for the example of a double quantum dot. We will make use of the recent observation ${ }^{14-16}$ that noise measurements in the nonlinear Coulomb-blockade regime can be used to obtain spectroscopic information of excited levels in a quantum dot. This scheme will be adapted to a double quantum dot, and we will show that the important interaction parameter $\phi$ (containing information about the entanglement) $)^{7,8}$ enters the Fano factor (i.e., the ratio of noise power to current) in this regime. This kind of noise spectroscopy can be used to directly extract $\phi$ from experimentally observable quantities. We extend a previous discussion ${ }^{17}$ of the low-frequency shot noise of a double-dot system by an analysis of the full counting statistics of the transferred charge. This allows us to identify directly the interaction parameter by a noise measurement. One main result is obtained in the transport regime, in which predominantly only one electron occupies the lowest double-dot level due to the Coulomb interaction. Thermal activation leads to tunneling events through excited twoelectron states, which result (under the conditions discussed below) in super-Poissonian shot noise characterized by a Fano factor

$$
F(\phi)=1+\phi^{2} \text {. }
$$

Hence, a measurement of the noise in this regime allows a direct determination of the interaction parameter $\phi$.

\section{MODEL AND THEORETICAL METHODS}

We now turn to a description of the model and the method. With maximally two electrons occupying the double dot and no magnetic field lifting the spin degeneracy, the following Hund-Mulliken eigenstates of the double quantum dot are possible (for a more detailed description, see Refs. 4 and 8): the zero-electron state $|0\rangle$, two twofold degenerate one-electron states $|+\rangle$ (symmetric) and $|-\rangle$ (antisymmetric), four two-electron states with one electron per dot, the singlet $|S\rangle$, and the threefold degenerate triplet $|T\rangle$. There are two other singlet states that have a significantly higher energy due to the large on-site Coulomb interaction, which do not have a physical effect in the regime studied (but are included in the numerical calculations).

We consider a (longitudinal) double dot coupled to leads, which are modeled as a Fermi sea. The tunnel coupling leads to a tunneling amplitude $t_{\alpha}$, which measures the overlap between the orbital state and the lead wave function in terminal $\alpha$. We assume that $V$ is the applied bias voltage and that $\mu_{L}=e V / 2\left(\mu_{R}=-e V / 2\right)$ are the chemical potentials of the left and right leads. There is an additional lead with capacitive coupling to the double dot characterized by the gate voltage $V_{g}$ that shifts the potential on the dot. When one electron is on the dot, it can be in two possible states: Its wave function is spread either symmetrically or antisymmetrically over the two dots. The symmetric state has a lower eigenenergy, and the energy difference between the two one-electron states depends on the interdot tunneling amplitude $t_{0}$, describing the potential barrier between the two dots. Including the energy contribution of the gate voltage, the eigenenergies of these states can be calculated as $E_{ \pm}=e V_{g} \mp t_{0}$. Adding a second electron, we first have to overcome the gate voltage again, and additionally the repulsive Coulomb interaction be- 
tween the two electrons leads to a spin-dependent splitting of the eigenenergies. For the singlet state $|S\rangle$ we obtain the energy $E_{S}=2 e V_{g}+u_{12}-J$, and for the (threefold degenerate) triplet state $|T\rangle$ the energy $E_{T}=2 e V_{g}+u_{12}$. Here, $u_{12}$ is the (interdot) electron-electron repulsion and $J=4 t_{0}^{2} / u_{H}$ is the Heisenberg exchange parameter that characterizes the Heisenberg interaction between the two spins, $H_{\text {spin }}$ $=J \mathbf{S}_{1} \cdot \mathbf{S}_{2} ; u_{H}$ is the on-site Coulomb repulsion. Because $J$ is positive, the singlet state has a lower energy than the triplet state.

The singlet state can be expressed in terms of the creation operators $d_{n}^{\dagger}, n= \pm$, which create an (anti)symmetric electron state

$$
|S\rangle=\frac{1}{\sqrt{1+\phi^{2}}}\left(d_{+\uparrow}^{\dagger} d_{+\downarrow}^{\dagger}-\phi d_{-\uparrow}^{\dagger} d_{-\downarrow}^{\dagger}\right)|0\rangle .
$$

The interaction parameter $\phi$ describes the competition between the kinetic-energy gain and the Coulomb repulsion. In the Hund-Mulliken model, $\phi=\sqrt{1+16 t_{0}^{2} / u_{H}^{2}}-4 t_{0} / u_{H}$ is determined by the interdot tunneling amplitude $t_{0}$ and the on-site Coulomb repulsion $u_{H}{ }^{4}$ In general, the dependence of $\phi$ on the microscopic parameters may have a different form; however, we will always use this definition for the quantitative plots below. It is important to stress that the results obtained below are largely independent of the precise dependence of $\phi$ on the details of the quantum dot. Hence, the noise features we find can be used as an additional test of the applicability of the model calculation of Ref. 4 to realistic quantum dots.

We describe transport through the double dot in the sequential-tunneling approximation using the master equation

$$
\frac{d P_{i}}{d t}=\sum_{j}\left(\Gamma_{j i} P_{j}-\Gamma_{i j} P_{i}\right) .
$$

Here, $i$ and $j$ label the available states of the quantum dot, including the number of electrons on the dot, the orbital index, and the spin. $P_{i}$ is the occupation probability of state $i$, and $\Gamma_{i j}$ is the tunneling rate from state $i$ to state $j$. The rates are given by

$$
\Gamma_{j i}=\gamma_{L} m_{i j} f\left[\sigma_{i j}\left(\epsilon_{i j}-\mu_{L}\right)\right]+\gamma_{R} m_{i j} f\left[\sigma_{i j}\left(\epsilon_{i j}-\mu_{R}\right)\right] .
$$

Here, $\gamma_{L / R}=\nu_{L / R}\left|t_{L / R}\right|^{2} / \pi$ is the bare tunneling rate to the left (right) terminal, and $\nu_{L / R}$ is the density of states in the leads. The matrix elements $m_{i j}$ depend on the orbital and the spin state of the dot. ${ }^{8}$ The occupation in the leads is determined by the Fermi functions $f(\epsilon)=1 /\left[\exp \left(\epsilon / k_{B} T\right)+1\right]$, the direction of tunneling is defined by $\sigma_{i j}= \pm 1$, and the energy difference is defined by $\epsilon_{i j}=E_{i}-E_{j}$. We note here that the interaction parameter $\phi$ enters the calculations through the matrix elements $m_{i j}$ of the singlet state. For a more detailed discussion of these tunneling rates, we refer to Ref. 8. For later use, we express the master equation [Eq. (3)] in matrix form, $d \mathbf{P} / d t=\mathbf{M P}$, where $M_{i j}=\Gamma_{j i}$ for $i \neq j$ and $M_{i i}=-\Sigma_{j \neq i} \Gamma_{i j}$.

From the master equation, we obtain the noise. ${ }^{18-22}$ The quantity characterizing the current noise is the Fano factor $F=S / 2 e I$. It measures how far the distribution of the tunneling events differs from the random Poissonian distribution:
For Poissonian noise, when the tunneling events are uncorrelated, $F=1$. A Fano factor $F<1$ can be due to anticorrelations (e.g., the Pauli principle). We will be mostly interested below in super-Poissonian noise, $F>1$, and we will show that here it is due to correlated transport cycles resulting from a blockade of open channels. By a transport cycle, we mean the following process: ${ }^{15}$ Suppose the system is initially in its ground state $A$, and another state $B$ (which would be available for tunneling according to its energy) is blocked due to Coulomb interaction. When this electron in $A$ tunnels out of the dot due to a thermal excitation, both the states $A$ and $B$ are available for electrons to tunnel into. A cycle is occurring when a sequence of electrons tunnels through state $B$ before an electron enters the ground state $A$ and blocks the transport through the dot again. This cycle leads to a correlated transfer of a number of charges given by the number electrons tunneling through the excited state $B$. Hence, that effective charge transferred in one cycle is larger than 1 and leads to an increased Fano factor.

The above picture is based on the sequential-tunneling approximation. The parameters have to be chosen such that cotunneling processes can be neglected. This is experimentally possible since the current due to cotunneling processes is generally of the order of $t^{4} / \Delta$, where $t$ is the tunneling amplitude and $\Delta$ is the excitation energy in the virtual intermediate state. In our case, the current in the most interesting regime is proportional to $t^{2} x$, where $x$ is exponentially small in $\Delta / k_{B} T$. Since the tunneling amplitude $t$ can, in principle, be made arbitrarily small, sequential tunneling becomes the dominant process. Recently, it was shown that noise spectroscopy deep inside the Coulomb-blockade regime is possible ${ }^{23-26}$ and in agreement with the sequential-tunneling description.

\section{CURRENT AND FANO FACTOR}

The results of our calculation are summarized in Fig. 1, which shows the average current (top panel) and the Fano factor (bottom panel) as a function of the bias voltage $V$ and the gate voltage $V_{g}$. Considering the current, the most visible feature is the Coulomb-blockade diamond (green in Fig. 1). As long as the bias voltage is lower than the energy difference between the energetically lowest state and the next state with more or less one electron, i.e., $e V<E_{i}\left(V_{g}\right)-E_{j}\left(V_{g}\right)$, with $i$ and $j$ being two states differing by one electron, electron tunneling is exponentially suppressed. In our model, we have three such blockade regimes with 0,1 , and 2 electrons in the dot for zero bias voltage. The two parts of Fig. 1 show the current and the Fano factor for the most interesting of these blockade regions, the one-electron blockade regime. We will concentrate our discussion on this region since most interesting features can be discussed here.

We first look at the energies of the states in the blockade regime as a function of the gate voltage (see Fig. 2). The capital letters in Figs. 1 and 2 correspond to these blockade regions, and we will now show that the features in the Fano factor can be explained by the energy plot for the states in this region. We first consider the regions A. They are both characterized by a Fano factor $F=1$, typical tunneling pro- 

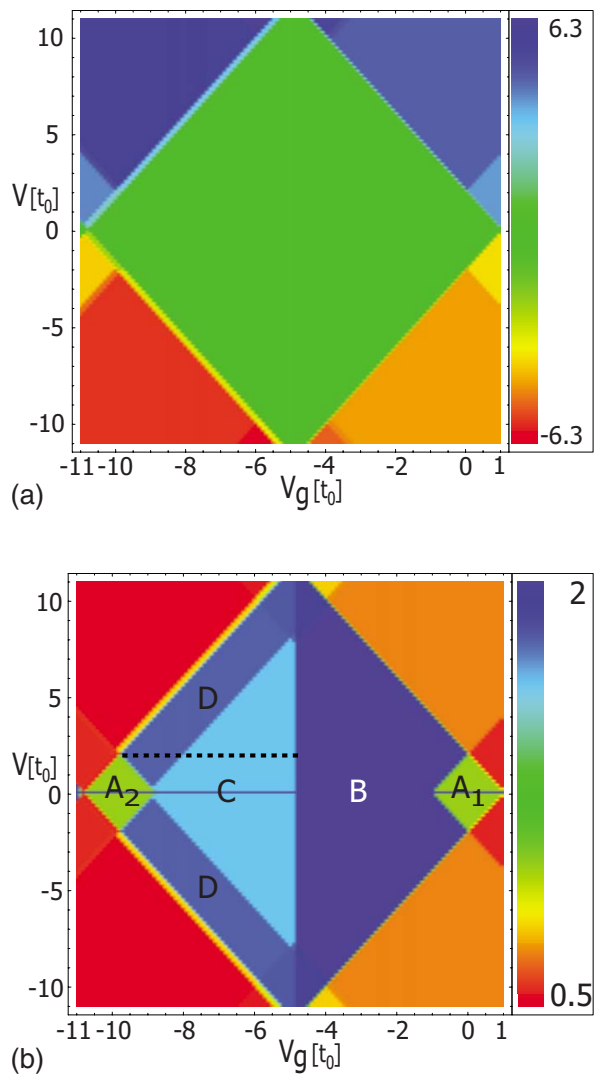

FIG. 1. (Color online) Current (top) and Fano factor (bottom) in the one-electron blockade regime as a function of gate voltage $V_{g}$ and bias voltage $V$. The current is given in units of $e \gamma$. Note the various plateaus in the Fano factor within the blockade regime, which are invisible in the current. The letters label the subregions as referred to in the main text. The dashed black line in the bottom plot indicates the line along which Figs. 3 and 4 are plotted. In this plot, we use the parameters $u_{H}=17 t_{0}, u_{12}=10 t_{0}, \gamma_{L}=\gamma_{R}=\gamma$, and $k_{B} T$ $=0.01 t_{0}\left(\right.$ and hence $J=0.24 t_{0}$ and $\left.\phi=0.79\right)$.

cesses through a simple two-level system. Indeed, Fig. 2 shows that the first excited states differ in electron number. In $A_{1}$, the excited state is $|0\rangle$; in $A_{2}$, the excited state is $|S\rangle$. Lowering the gate voltage, we come to region $\mathrm{B}$, where the first excited state is the state $|-\rangle$, i.e., a state with one elec-

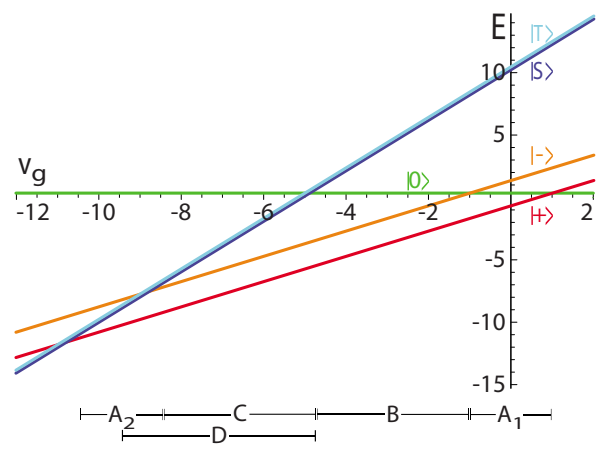

FIG. 2. (Color online) Energy of the states as a function of gate voltage $V_{g}$ in units of $t_{0}$. The capital letters correspond to the regions in Fig. 1. The parameters are chosen like in Fig. 1.

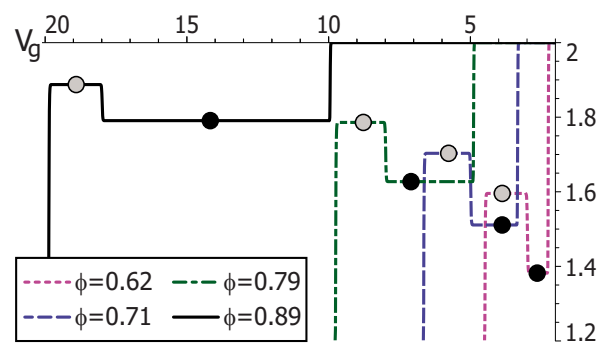

FIG. 3. (Color online) Fano factor for different values of $\phi$ in regions $\mathrm{C}$ and $\mathrm{D}$ as a function of gate voltage (in units of $t_{0} / e$ ). The bias voltage is $e V=2 t_{0}$ and $k_{B} T=0.01 t_{0}$. Furthermore, the ratio $u_{12} / u_{H}=0.6$ is kept fixed, such that the different plots can be easily achieved in experiment by changing the interdot tunneling rate $t_{0}$. The parameters are chosen as $\left(\phi ; J / t_{0}, u_{12} / t_{0}\right)=(0.62 ; 0.5,5)$, $(0.71 ; 0.34,7),(0.79 ; 0.24,10)$, and $(0.89 ; 0.12,20)$ from right to left. The plateau heights, indicated by the black (region C) and gray (region D) dots, follow from the analytic expressions [Eqs. (1) and (7)].

tron as well. Thus, the description in terms of a single state, which can be occupied or not, does not hold any more, and we observe an increase in the Fano factor to $F=2$. In this region, the state $|0\rangle$ is still the first state available for tunneling, but the blocked state $|-\rangle$ is energetically lower. Continuing to region $\mathrm{C}$, the energies of the two-electron states become lower than the zero-electron state. So, the first available excited state is the singlet $|S\rangle$. The difference between regions $\mathrm{C}$ and $\mathrm{D}$ is somewhat more complicated. Since the difference $E_{S}-E_{-}$is smaller than $E_{S}-E_{+}$, the state $|+\rangle$ still blocks the dot if it is occupied. After a thermal excitation to state $|S\rangle$, electrons tunnel through states $|S\rangle$ and $|-\rangle$ until an electron occupies state $|+\rangle$ again and blocks the dot. In regions $\mathrm{C}$ and $\mathrm{D}$, the Fano factor depends on the parameter $\phi$ through the prefactor of the singlet states [see Eq. (2)]. The $\phi$ dependence is illustrated in Fig. 3 by varying the parameter $u_{H} / t_{0}$. As can be seen, the positions of the steps change simultaneously with the plateau height. However, the height depends only on the interaction parameter, as we have checked by numerically varying $u_{H}$ and $\phi$ independently. We will derive below analytic expressions for the Fano factor, which confirm this behavior.

The effect of temperature will be to wash out the sharp steps and plateaus. This is illustrated in Fig. 4. Increasing the temperature to a value of the order of the level splitting first the sharp steps are washed out. The plateaus C and D, however, remains still visible and the values are still given, at least approximately, by the low temperature results. Finally, the temperature leads to a vanishing of the plateaus, which makes it difficult to extract the interaction parameter $\phi$ from the Fano factor.

\section{COUNTING STATISTICS}

A more detailed view of the transport characteristic can be obtained by looking at the full counting statistics. ${ }^{21}$ The aim here is to find the cumulant generating function (CGF) $\mathcal{S}(\chi)$, which is related to the probability $P(N)$ of $N$ charges passing 


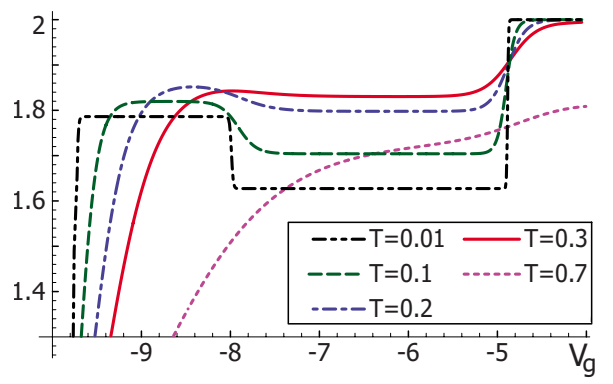

FIG. 4. (Color online) Fano factor as a function of gate voltage (in units of $t_{0} / e$ ) at various temperatures for $\phi=0.79, u_{H}=17 t_{0}$, and $u_{12}=10 t_{0}$. The steps in the Fano factor are washed out by the temperature; however, the noise remains super-Poissonian in the whole regime shown.

through the system during the measurement time $t_{M}$ by $\exp \mathcal{S}(\chi)=\Sigma_{N} P(N) e^{i N \chi}$. The knowledge of $\mathcal{S}(\chi)$ is equivalent to the knowledge of all the cumulants of the system according to $C_{k}=\left.\left(-i \partial / \partial_{\chi}\right)^{k} \mathcal{S}(\chi)\right|_{\chi=0}$, such as current (first cumulant) and noise (second cumulant), $I=e C_{1} / t_{M}$ and $S=2 e^{2} C_{2} / t_{M}$, respectively (where $e$ is the electron charge). The simplest way to evaluate the counting statistics in this case is to use the method described in Ref. 21. We choose (without loss of generality) to count the charges in the left lead. Therefore, we have to change the left tunneling rates in the off-diagonal elements of the transport matrix $\mathbf{M}: \Gamma_{L} \rightarrow \Gamma_{L} \exp (i \chi)$. The smallest eigenvalue of $\mathbf{M}$, which we denote by $\lambda_{0}(\chi)$, determines the CGF as $\mathcal{S}(\chi)=-t_{M} \lambda_{0}(\chi)$.

If we restrict our calculations to a certain region and thus reduce the number of involved states, it is possible to get an analytical expression for the Fano factor. ${ }^{15}$ For region $\mathrm{C}$, we include the one-electron states $|+\rangle$ and $|-\rangle$, and the twoelectron singlet $|S\rangle$. Here, the relevant energy differences are both smaller than the bias: $E_{S}-E_{-}<E_{S}-E_{+}<e V / 2$. Therefore, both Fermi functions for the tunneling processes are exponentially suppressed, but one is much bigger than the other,

$$
1 \gg f\left(E_{S}-E_{+}-e V / 2\right) \gg f\left(E_{S}-E_{-}-e V / 2\right) .
$$

Therefore, we neglect $f\left(E_{S}-E_{-}-e V / 2\right)$ and take the rates to lowest order in the parameter $x \equiv f\left(E_{S}-E_{+}-e V / 2\right)$. Then, the tunneling rates are

$$
\begin{gathered}
\Gamma_{+S} \approx \frac{1}{1+\phi^{2}} \gamma_{L} x, \quad \Gamma_{S+} \approx \frac{2}{1+\phi^{2}}\left(\gamma_{L}+\gamma_{R}\right), \\
\Gamma_{-S} \approx \frac{\phi^{2}}{1+\phi^{2}} \gamma_{L}, \quad \Gamma_{S-} \approx \frac{2 \phi^{2}}{1+\phi^{2}}\left(\gamma_{L}+\gamma_{R}\right) .
\end{gathered}
$$

Here, $\gamma_{L / R}$ are the bare tunneling rates for the left/right leads. Without loss of generality, we will count the charges in the left lead, which means we have to replace $\gamma_{L} \leftrightarrow \gamma_{L} \exp ( \pm i \chi)$ (the sign depending on whether an electron enters or leaves the dot) in the off-diagonal elements of the matrix M. The counting statistics is obtained from the smallest eigenvalue $\lambda_{0}$, which we determine to lowest order in $x$. The result is

$$
S(\chi)=-x \frac{\gamma_{L} \gamma_{R}\left(1+\phi^{2}\right)}{\gamma_{R}\left(1+\phi^{2}\right)-\gamma_{L}} \frac{e^{i \chi}-1}{1-p(\phi) e^{i \chi}},
$$

where $p(\phi)=\phi^{2} \gamma_{R} /\left[\gamma_{L}+\gamma_{R}\left(1+\phi^{2}\right)\right]$. Finally, we obtain the Fano factor

$$
F(\phi)=\frac{1+p(\phi)}{1-p(\phi)}=1+\phi^{2} \frac{2 \gamma_{R}}{\gamma_{L}+\gamma_{R}},
$$

which is independent of the bias voltage as long as we are in the one-electron Coulomb blockade regime. For a symmetric structure with $\gamma_{L}=\gamma_{R}$, we obtain Eq. (1).

A similar result may be obtained for region D in Fig. 1. Here, the situation is a bit more complicated since the lowest-lying triplet state also plays a role; thus, the problem involves four states. In the same way as before, we neglect $f\left(E_{T}-E_{ \pm}-e V / 2\right)$ since the transition between $|S\rangle \leftrightarrow|+\rangle$ is the least suppressed. Assuming that the temperature is much smaller than the singlet-triplet splitting, we calculate the series expansion for the Fano factor (with symmetric leads) to be

$$
F(\phi)=\frac{2+17 \phi^{2}+32 \phi^{4}+16 \phi^{6}}{2+11 \phi^{2}+16 \phi^{4}+3 \phi^{6}} .
$$

Both these results, Eqs. (1) and (7), are in agreement with our numerical calculations, as shown in Fig. 3.

\section{CONCLUSIONS}

In conclusion, we have shown that noise measurements in the Coulomb-blockade regime of a double quantum dot can reveal interesting information about the quantum correlations created during tunneling. In particular, we have shown that the Fano factor in the one-electron blockade region is superPoissonian and can be used to determine the interaction parameter $\phi$. This allows us to measure the degree of entanglement of two electrons in the double quantum dot.

\section{ACKNOWLEDGMENTS}

We would like to thank D. Loss for useful discussions. This work was financially supported by the Swiss National Science Foundation, by the NCCR Nanoscience, by the Deutsche Forschungsgemeinschaft within the SFB 513 and the Priority Program Semiconductor Spintronics, and by the Landesstiftung Baden-Württemberg within the Kompetenznetzwerk Funktionelle Nanostrukturen. 
${ }^{1}$ Ya. M. Blanter and M. Büttiker, Phys. Rep. 336, 1 (2000).

${ }^{2}$ Quantum Noise in Mesoscopic Physics, edited by Yu. V. Nazarov (Kluwer, Dordrecht, 2003).

${ }^{3}$ D. Loss and D. P. DiVincenzo, Phys. Rev. A 57, 120 (1998).

${ }^{4}$ G. Burkard, D. Loss, and D. P. DiVincenzo, Phys. Rev. B 59, 2070 (1999).

${ }^{5}$ R. Ziegler, C. Bruder, and H. Schoeller, Phys. Rev. B 62, 1961 (2000).

${ }^{6}$ W. G. van der Wiel, S. De Franceschi, J. M. Elzerman, T. Fujisawa, S. Tarucha, and L. P. Kouwenhoven, Rev. Mod. Phys. 75, 1 (2003).

${ }^{7}$ V. N. Golovach and D. Loss, Europhys. Lett. 62, 83 (2003).

${ }^{8}$ V. N. Golovach and D. Loss, Phys. Rev. B 69, 245327 (2004).

${ }^{9}$ M. R. Graber, W. A. Coish, C. Hoffmann, M. Weiss, J. Furer, S. Oberholzer, D. Loss, and C. Schönenberger, Phys. Rev. B 74, 075427 (2006).

${ }^{10}$ B. R. Bulka and T. Kostyrko, Phys. Rev. B 70, 205333 (2004).

${ }^{11}$ L. DiCarlo, H. J. Lynch, A. C. Johnson, L. I. Childress, K. Crockett, C. M. Marcus, M. P. Hanson, and A. C. Gossard, Phys. Rev. Lett. 92, 226801 (2004).

${ }^{12}$ T. Hatano, M. Stopa, T. Yamaguchi, T. Ota, K. Yamada, and S. Tarucha, Phys. Rev. Lett. 93, 066806 (2004).

${ }^{13}$ B. Wunsch, M. Braun, J. König, and D. Pfannkuche, Phys. Rev. B 72, 205319 (2005).

${ }^{14}$ A. Cottet and W. Belzig, Europhys. Lett. 66, 405 (2004); A. Cottet, W. Belzig, and C. Bruder, Phys. Rev. Lett. 92, 206801 (2004).

${ }^{15}$ W. Belzig, Phys. Rev. B 71, 161301(R) (2005).
${ }^{16}$ A. Cottet, W. Belzig, and C. Bruder, Phys. Rev. B 70, 115315 (2004).

${ }^{17}$ J. Aghassi, A. Thielmann, M. H. Hettler, and G. Schön, Phys. Rev. B 73, 195323 (2006).

${ }^{18}$ A. N. Korotkov, Phys. Rev. B 49, 10381 (1994).

${ }^{19}$ S. Hershfield, J. H. Davies, P. Hyldgaard, C. J. Stanton, and J. W. Wilkins, Phys. Rev. B 47, 1967 (1993).

${ }^{20}$ U. Hanke, Yu. M. Galperin, K. A. Chao, and N. Zou, Phys. Rev. B 48, 17209 (1993).

${ }^{21}$ D. A. Bagrets and Yu. V. Nazarov, Phys. Rev. B 67, 085316 (2003).

${ }^{22}$ B. R. Bulka, J. Martinek, G. Michalek, and J. Barnas, Phys. Rev. B 60, 12246 (1999); B. R. Bulka, ibid. 62, 1186 (2000).

${ }^{23}$ S. Gustavsson, R. Leturcq, B. Simovic, R. Schleser, T. Ihn, P. Studerus, K. Ensslin, D. C. Driscoll, and A. C. Gossard, Phys. Rev. Lett. 96, 076605 (2006); S. Gustavsson, R. Leturcq, B. Simovic, R. Schleser, P. Studerus, T. Ihn, K. Ensslin, D. C. Driscoll, and A. C. Gossard, Phys. Rev. B 74, 195305 (2006).

${ }^{24}$ T. Fujisawa, T. Hayashi, R. Tomita, and Y. Hirayama, Science 312, 1634 (2006).

${ }^{25}$ E. V. Sukhorukov, A. N. Jordan, S. Gustavsson, R. Leturcq, T. Ihn, and K. Ensslin, Nat. Phys. 3, 243 (2007).

${ }^{26}$ Y. Zhang, L. DiCarlo, D. T. McClure, M. Yamamoto, S. Tarucha, C. M. Marcus, M. P. Hanson, and A. C. Gossard, Phys. Rev. Lett. 99, 036603 (2007); D. T. McClure, L. DiCarlo, Y. Zhang, H.-A. Engel, C. M. Marcus, M. P. Hanson, and A. C. Gossard, ibid. 98, 056801 (2007). 